

Physical Activity and Nutrition I 2021;25(4):054-058

Received: 2021/11/11, Revised: 2021/12/08

Accepted: 2021/12/30, Published: 2021/12/31

@2021 Wonchung Lim et al.; Licence Physical Activity and Nutrition. This is an open access article distributed under the terms of the creative commons attribution license (https:/l creativecommons.org/licenses/by-nc/2.0/), which permits unrestricted use, distribution, and reproduction in any medium, provided the orginal work is properly cited.

*Corresponding author: Wonchung Lim, Ph.D.

Department of Sports Medicine, College of Health Science, Cheongju University, Cheongju 28503, Republic of Korea.

Tel: +82-43-229-8629

E-mail: wonchlim@gmail.com

(๑2021 The Korean Society for Exercise Nutrition

[Purpose] Deleted in breast cancer 1 (DBC1) ablation causes obesity, and stearoyl-CoA desaturase 1 (SCD1) induces the biosynthesis of monounsaturated fatty acids. This study examined whether voluntary wheel running (WWR) alters SCD-1 and DBC1 protein levels in the liver of leptin-deficient ob/ob mice.

[Methods] Twenty-five Ob/Ob mice were divided into two groups (ob/ob-Sed and ob/ob-Ex). The expression of DBC1 and SCD1 in the mouse liver was determined using western blotting.

[Results] After 10 weeks, WWR significantly reduced body weight without affecting the fatty acid synthase and CD36 protein levels. The average daily running distance was $4.0 \pm 1.0 \mathrm{~km} /$ day. This improvement was associated with changes in the hepatic SCD1 and DBC1 levels. Hepatic SCD-1 protein levels increased significantly, and DBC1 protein levels decreased in ob/ ob-Sed animals. On the other hand, WWR inhibited the obesity-induced increase in SCD1 expression and impaired the obesity-induced decrease in DBC1 expression in the liver of leptin-deficient ob/ob mice.

[Conclusion] This is the first study showing that WWR has strong effects on hepatic SCD1 and DBC1 in ob/ ob mice, and provides key insights into the effects of exercise on obesity.

[Keywords] exercise, obesity, deleted in breast cancer 1 , stearoyl-CoA desaturase 1 , body weight, ob/ob mice

\section{Voluntary exercise training improves body weight of leptin-deficient ob/ ob mice by altering hepatic stearoyl- CoA desaturase 1 and deleted in breast cancer 1 protein levels}

\author{
Wonchung Lim ${ }^{1 *}$ / Moon-Hyon Hwang ${ }^{2}$ / Chounghun Kang ${ }^{3}$ / \\ So Yeon $\mathrm{Kim}^{4}$ / Hyeseong $\mathrm{Cho}^{5}$ \\ 1. Department of Sports Medicine, Cheongju University, Cheongju, Republic of Korea \\ 2. Division of Health \& Kinesiology, Incheon National University, Incheon, Republic of Korea \\ 3. Department of Physical Education, Inha University, Republic of Korea \\ 4. Department of Dental Hygiene, Cheongiu University, Cheongju, Republic of Korea \\ 5. Department of Biochemistry and Molecular Biology, Ajou University School of Medicine, Suwon, Republic of \\ Korea
}

\section{INTRODUCTION}

Obesity is a growing health problem worldwide ${ }^{1}$ and is associated with insulin resistance and type 2 diabetes ${ }^{2,3}$. An imbalance between lipid storage and oxidation in the liver is important for the development of obesity ${ }^{4}$. Stearoyl-CoA desaturase (SCD) is a rate-limiting enzyme that catalyzes the biosynthesis of monounsaturated fatty acids and is a key regulatory factor in body adiposity ${ }^{5}$. Loss of SCD1 function protects mice against adiposity ${ }^{6}$. Moreover, downregulation of SCD1 is an important component of the metabolic action of leptin in the liver ${ }^{7}$.

Exercise is widely accepted as a treatment for obesity. Regular exercise helps reduce body weight and fat levels, maintain body weight and fat levels, and improve the metabolic fitness of obese people ${ }^{1}$. Voluntary exercise has beneficial effects beyond those of simple muscle contractions. These include powerful neural feedforward signals to the cardiovascular, respiratory, metabolic, and hormonal systems along with neural feedback from contracting skeletal muscles ${ }^{8}$. Exercise-induced enhancement of substrate oxidation is essential for the treatment of obesity because this condition is associated with impaired skeletal muscle lipid utilization ${ }^{9}$. Increased SCD1 activity via exercise might be protective against excessive weight gain ${ }^{10}$. A decrease in SCD-1 gene expression is an important mechanism that converges with the proper regulation of lipid metabolism by the liver ${ }^{11}$. However, the effects of voluntary wheel running (VWR) on SCD1 expression in ob/ob mouse livers are unknown. This study examined whether VWR alters SCD-1 and DBC1 protein levels in the livers of leptin-deficient ob/ob mice.

\section{METHODS}

\section{Animals}

Mice were divided into the wild-type sedentary group (WT-SD; $n=8$ ), 
ob/ob sedentary group (ob-SD; n=15), and ob/ob + VWR group (ob-EX group; $\mathrm{n}=10$ ). The WT-SD and ob-SD groups did not perform any exercises. All protocols were approved by the Chungbuk National University Institutional Animal Care and Use Committee (CBNUA-1357-20-01). Voluntary wheel running (VWR) is an aerobic exercise. Five-weekold male ob/ob mice (C57BL/6J background strain) were obtained from Taconic (USA). Ob/ob mice $(n=10)$ were housed individually in cages with free access to running wheels to monitor daily running activity (Lafayette Instruments Neuroscience, USA) for a period of 9 weeks. The average daily running distance was $4 \pm 1.0 \mathrm{~km} /$ day.



Voluntary wheel running system (aerobic exercise)

Figure 1. Voluntary wheel running system.

\section{Western blot analysis}

Proteins were isolated using lysis buffer $(150 \mathrm{mM} \mathrm{NaCl}$, $50 \mathrm{mM}$ Tris-HCl, $5 \mathrm{mM}$ EDTA, 1\% Nonidet P-40, 0.5\% deoxycholate, and $1 \%$ SDS) with a protease inhibitor cocktail (Sigma, St. Louis, MO, USA) on ice for $1 \mathrm{~h}$ and then centrifuged for $20 \mathrm{~min}$ at $13,000 \times \mathrm{g}$. The supernatant was collected, and protein concentrations were measured using the Bradford method (Bio-Rad, Hercules, CA, USA). Proteins were dissolved in the sample buffer and boiled for 5 min prior to loading onto an acrylamide gel. After SDSPAGE, the proteins were transferred to a polyvinylidene difluoride membrane and blocked with 5\% non-fat dry milk in Tris-buffered saline containing $0.1 \%$ Tween-20 (TBST) for $60 \mathrm{~min}$ at room temperature. The membranes were incubated with the primary antibody for $2 \mathrm{~h}$ at room temperature. Equal lane loading was assessed using an actin monoclonal antibody (Sigma-Aldrich). After washing with TBST, the blots were incubated with a 1:5000 dilution of horseradish peroxidase-conjugated secondary antibody (Invitrogen, Grand Island, NY, USA) and washed again three times with TBST. The transferred proteins were visualized using an enhanced chemiluminescence detection kit (Amersham Pharmacia Biotech).

\section{Statistical analysis}

Data are expressed as mean $\pm \mathrm{SD}$, and statistical analysis for single comparisons was performed using the Student's $t$-test. The criterion for statistical significance was set at $P<$ 0.05 .

\section{RESULTS}

Effects of VWR on body weights in wild-type (WT) and ob/ob mice.

Body weight increased significantly over the 9 -week period in all groups. At week 9, the body weight remained higher in both ob/ob mouse groups than in the WT control group. However, with voluntary wheel running, body weight was lower in ob/ob mice than in sedentary ob/ob mice (WT$\mathrm{SD}$ vs. ob-SD $* \mathrm{P}<0.05, * * \mathrm{P}<0.001$, ob-SD vs. ob-EX \# $\mathrm{P}$ $<0.05$, \#\# $\mathrm{P}<0.001$. Figure 2).



Figure 2. Effects of VWR on body weights in wild-type (WT) and ob/ob mice. Values are reported as mean \pm SD for mice per group. WT-SD vs ob-SD * $P<0.05,{ }^{* *} \mathrm{P}<0.001$, ob-SD vs ob-EX \# $\mathrm{P}<$ 0.05 , \#\# $\mathrm{P}<0.001$.

Obesity induces SCD1 expression, and voluntary exercise inhibits obesity induction of SCD1 expression in the liver of leptin-deficient ob/ob mice.

SCD1 is induced by obesity and has been implicated in nonalcoholic fatty liver disease ${ }^{12,13}$. However, there are no reports of SCD1 behavior in response to voluntary exercise in the livers of leptin-deficient ob/ob mice. The protein expression of SCD1 was significantly increased in the livers of ob/ob mice compared with that in WT control mice. Voluntary exercise blocked obesity-induced increase in hepatic SCD1 protein expression (WT-SD vs. ob-SD $* \mathrm{P}<0.05$, ob$\mathrm{SD}$ vs. ob-EX \# $\mathrm{P}<0.05$. Figure 3 ).

Obesity reduces deleted in breast cancer 1 (DBC1) expression, and voluntary exercise impairs the obesity-induced decrease in Dbc1 expression in the livers of leptin-deficient ob/ob mice.

DBC1 limits the accumulation of adipose tissue fat and plays an important role in the development of metabolic syndrome ${ }^{14}$. Moreover, DBC1 ablation caused obesity by elevating SCD $1{ }^{15}$ levels. Therefore, $\mathrm{DBC} 1$ protein levels were measured to determine whether the inhibition of SCD1 expression by voluntary exercise could be explained by DBC1. As shown in Figure 4, DBC1 protein levels were decreased in the livers of ob/ob mice; these effects were attenuated by VWR (WT-SD vs. ob-SD *P $<0.05$, ob-SD vs. ob-EX \# P $<$ 


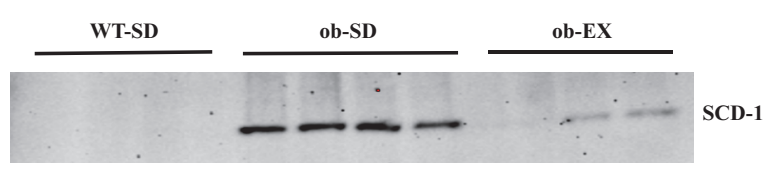

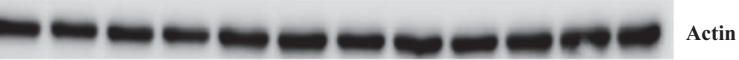

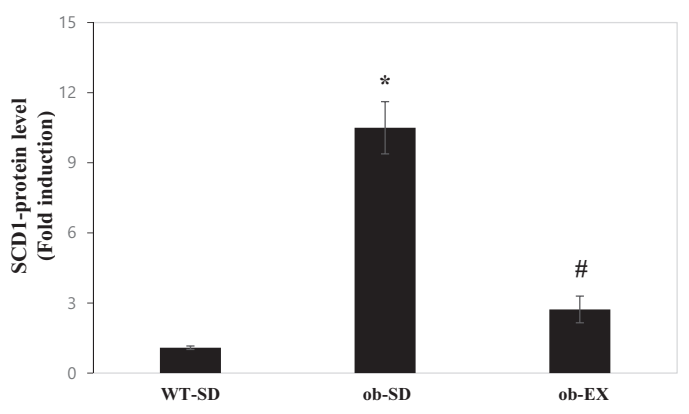

Figure 3. Obesity induces SCD1 expression, and voluntary exercise inhibits obesity induction of SCD1 expression in the liver of leptin-deficient ob/ob mice. Values are reported as mean \pm SD for mice per group. WT-SD vs ob-SD *P $<0.05$, ob-SD vs ob-EX, \# P $<0.05$.
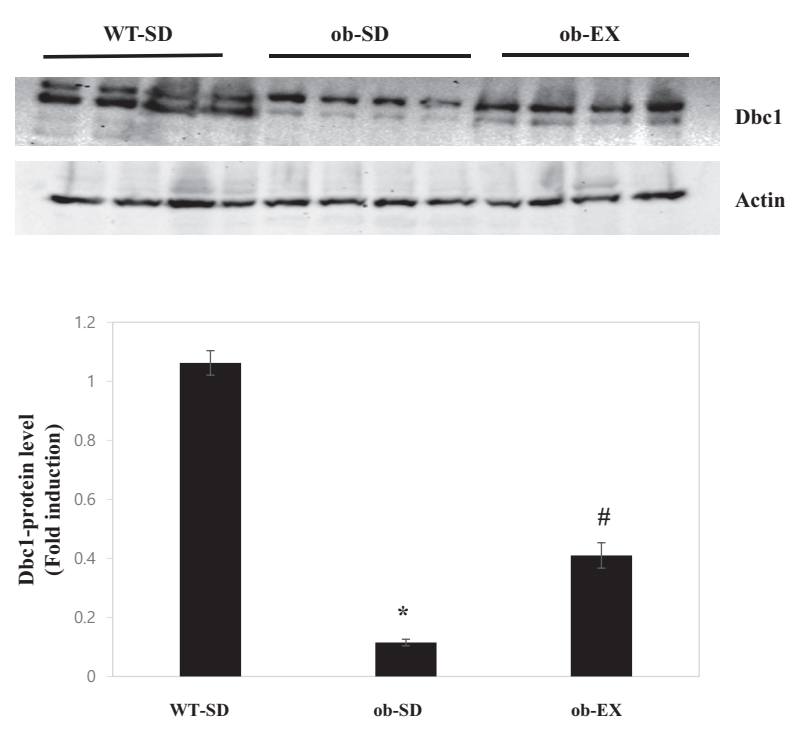

Figure 4. Obesity reduces deleted in breast cancer 1 (DBC1) expression and voluntary exercise impairs the obesity-induced decrease of $\mathrm{DBC} 1$ expression in the liver of leptin-deficient ob/ob mice. Values are reported as mean \pm SD for mice per group. WTSD vs ob-SD *P $<0.05$, ob-SD vs ob-EX, \# $P<0.05$

0.05). These results suggest that DBC1 may be involved in voluntary exercise-promoted inhibition of obesity-induced $\mathrm{SCD} 1$ expression in the livers of ob/ob mice.

Effects of VWR on fatty acid synthase (FAS) and cluster determinant 36 (CD36) protein levels in the livers of leptin-deficient ob/ob mice.

FAS in the liver is important for de novo lipogeneis ${ }^{16}$. Induction of CD36 expression is closely associated with obe- sity ${ }^{17}$. This study examined the effects of voluntary exercise on FAS and CD36 protein expression levels. As shown in Figure 5, voluntary exercise did not affect the obesity-induction of FAS and CD36 expression in the livers of leptin-deficient ob/ob mice (WT-SD vs. ob-SD *P $<0.05$.).



Figure 5. Effects of VWR on fatty acid synthase (FAS) and cluster determinant 36 (CD36) protein levels in the livers of leptin-deficient ob/ob mice. Values are reported as mean \pm SD for mice per group. WT-SD vs ob-SD, ${ }^{*} \mathrm{P}<0.05$.

\section{DISCUSSION}

This study examined why voluntary exercise improved the body weight of leptin-deficient ob/ob mice. These results show that voluntary exercise inhibited obesity-induced SCD1 expression. In addition, VWR increased $\mathrm{DBC} 1$ protein levels without affecting FAS or CD36 protein levels.

Exercise training has many metabolic benefits, including plasma lipoprotein and triglyceride (TG) levels ${ }^{18}$, and glycemic control ${ }^{19}$. Voluntary aerobic exercise prevents ageand high-fat-induced vascular dysfunction, mitochondrial oxidative stress, and inflammation in mice, ${ }^{20}$ and improves motor function and spinal cord plasticity ${ }^{21}$. These benefits could be preserved in those who exercise throughout their lives. Therefore, an exercise program is an important intervention option for reducing risk factors associated with obesity. Many unknown exercise-mediated signaling networks remain to be identified. From this perspective, SCD1 could be the main target of voluntary exercise-induced signaling pathways.

The ob/ob leptin deficiency genetic model was selected because it represents a phenotype of severe obesity ${ }^{22}$. To the best of our knowledge, this is the first study to provide evidence that voluntary exercise inhibits hepatic SCD-1 expression in ob/ob mice. DBC1 levels were measured to gain insights into how SCD-1 may be affected by voluntary 
exercise training. Dbc1 ablation induces metabolic abnormalities mediated by SCD1 and hepatic sirtuin 1 (SIRT1) ${ }^{15}$. The deacetylase activity of SIRT1 induces SCD1 expression while DBC1 suppresses SCD1 by blocking SIRT1 ${ }^{15}$. In this system, obesity-induced decrease in $\mathrm{DBC} 1$ expression was attenuated by voluntary exercise. Cross-talk between voluntary exercise and SCD1 may involve an interaction between DBC1 and SIRT1. Voluntary aerobic exercise may affect the SCD1/DBC1/SIRT1 network, which may improve body weight in obese individuals. Further studies are required to elucidate this mechanism.

The FAS/SCD1 axis plays a critical role in TG accumulation $^{23}$. The finding that FAS and CD36 protein levels were unaffected by voluntary aerobic exercise in ob/ob mice is intriguing. This suggests that the body weight was higher in the ob/ob exercise group than in the WT control group. In this system, the main target of voluntary aerobic exercise is hepatic SCD1 but not FAS.

Until now, little attention has been paid to the role of voluntary aerobic exercise training in obesity-induced SCD1/ $\mathrm{DBC} 1$ signaling. These results indicate that voluntary exercise plays a critical role in hepatic $\mathrm{SCD} 1 / \mathrm{DBC} 1$ regulation and improves body weight in leptin-deficient ob/ob mice. This suggests that SCD1/DBC1 may be a key therapeutic target for obesity and diabetes.

\section{ACKNOWLEDGEMENTS}

This work was supported by the National Research Foundation of Korea (NRF-2018R1D1A1B07048750 and NRF2019S1A5A2A03051702) funded by the Korean government.

\section{REFERENCES}

1. Petridou A, Siopi A, Mougios V. Exercise in the management of obesity. Metabolism. 2019;92:163-9.

2. Azuma K, Heilbronn LK, Albu JB, Smith SR, Ravussin E, Kelley DE, Look AHEAD Adipose Research Group. Adipose tissue distribution in relation to insulin resistance in type 2 diabetes mellitus. Am J Physiol Endocrinol Metab. 2007;293:E435-42.

3. Bray GA, Jablonski KA, Fujimoto WY, Barrett-Connor E, Haffner $S$, Hanson RL, Hill JO, Hubbard V, Kriska A, Stamm E, Pi-Sunyer FX, Diabetes Prevention Program Research Group. Relation of central adiposity and body mass index to the development of diabetes in the diabetes prevention program. Am J Clin Nutr. 2008;87:1212-8.

4. Quiroga AD, Lehner R. Liver triacylglycerol lipases. Biochim Biophys Acta. 2012;1821:762-9.

5. Dobrzyn P, Ntambi JM, Dobrzyn A. Stearoyl-CoA desaturase: a novel control pint of lipid metabolism and insulin sensitivity. Eur J Lipid Sci Technol. 2008;110:93-100.

6. Ntambi JM, Miyazaki M, Stoehr JP, Lan H, Kendziorski CM, Yandell BS, Song Y, Cohen P, Friedman JM, Attie AD. Loss of stearoyl-CoA desaturase-1 function protects mice against adiposity. Proc Natl Acad Sci USA. 2002;99:11482-6.

7. Cohen P, Miyazaki M, Socci ND, Hagge-Greenberg A, Liedtke W,
Soukas AA, Sharma R, Hudgins LC, Ntambi JM, Friedman JM. Role for stearoyl-CoA desaturase-1 in leptin-mediated weight loss. Science. 2002;297:240-3.

8. Hawley JA, Hargreaves M, Joyner MJ, Zierath JR. Integrative biology of exercise. Cell. 2014;159:738-49.

9. O'Gorman DJ, Krook A. Exercise and the treatment of diabetes and obesity. Endocrinol Metab Clin North Am. 2008;37:887-903.

10. Rogowski MP, Flowers MT, Stamatikos AD, Ntambi JM, Paton CM. SCD1 activity in muscle increases triglyceride PUFA content, exercise capacity, and PPARס expression in mice. J Lipid Res. 2013;54:2636-46.

11. Yasari S, Prud'homme D, Wang D, Jankowski M, Levy E, Gutkowska J, Lavoie JM. Exercise training decreases hepatic SCD1 gene expression and protein content in rats. Mol Cell Biochem. 2010;335:291-9.

12. Zhu X, Bian H, Wang L, Sun X, Xu X, Yan H, Xia M, Chang X, Lu Y, Li Y, Xia P, Li X, Gao X. Berberine attenuates nonalcoholic hepatic steatosis through the ampk-srebp-1c-SCD1 pathway. Free Radic Biol Med. 2019;141:192-204.

13. Ortinau LC, Pickering RT, Nickelson KJ, Stromsdorfer KL, Naik CY, Haynes RA, Bauman DE, Rector RS, Fritsche KL, Perfield JW 2nd. Sterculic oil, a natural SCD1 inhibitor, improves glucose tolerance in obese ob/ob Mice. ISRN Endocrinol. 2012;2012:947323.

14. Escande C, Nin V, Pirtskhalava T, Chini CC, Tchkonia T, Kirkland $\mathrm{JL}$, Chini EN. Deleted in breast cancer 1 limits adipose tissue fat accumulation and plays a key role in the development of metabolic syndrome phenotype. Diabetes. 2015;64:12-22.

15. Qiang L, Kon N, Zhao W, Jiang L, Knight CM, Welch C, Pajvani $\mathrm{U}, \mathrm{Gu}$ W, Accili D. Hepatic sirT1-dependent gain of function of stearoyl-coa desaturase-1 conveys dysmetabolic and tumor progression functions. Cell Rep. 2015;11:1797-808.

16. Baldini SF, Wavelet C, Hainault I, Guinez C, Lefebvre T. The nutrient-dependent O-GIcNAc modification controls the expression of liver fatty acid synthase. J Mol Biol. 2016;428:3295-304.

17. Su T, Huang C, Yang C, Jiang T, Su J, Chen M, Fatima S, Gong R, Hu X, Bian Z, Liu Z, Kwan HY. Apigenin inhibits STAT3/CD36 signaling axis and reduces visceral obesity. Pharmacol Res. 2020; 152:104586.

18. Després JP, Lamarche B. Low-intensity endurance exercise training, plasma lipoproteins and the risk of coronary heart disease. $J$ Intern Med. 1994;236:7-22.

19. Boulé NG, Haddad E, Kenny GP, Wells GA, Sigal RJ. Effects of exercise on glycemic control and body mass in type 2 diabetes mellitus: a meta-analysis of controlled clinical trials. JAMA. 2001;286:1218-27.

20. Gioscia-Ryan RA, Clayton ZS, Zigler MC, Richey JJ, Cuevas LM, Rossman MJ, Battson ML, Ziemba BP, Hutton DA, VanDongen NS, Seals DR. Lifelong voluntary aerobic exercise prevents ageand Western diet-induced vascular dysfunction, mitochondrial oxidative stress and inflammation in mice. J Physiol. 2021;599:91125.

21. Zhang W, Yang B, Weng H, Liu T, Shi L, Yu P, So KF, Qu Y, Zhou L. Wheel running improves motor function and spinal cord plasticity in mice with genetic absence of the corticospinal tract. Front Cell Neurosci. 2019;13:106.

22. Roden M, Ludwig C, Nowotny P, Schneider B, Clodi M, Vierhapper $\mathrm{H}$, Roden A, WaldhäusI W. Relative hypoleptinemia in patients with type 1 and type 2 diabetes mellitus. Int J Obes Relat Metab 
Disord. 2000;24:976-81.

23. Zhang M, Sun W, Zhou M, Tang Y. MicroRNA-27a regulates hepat-

ic lipid metabolism and alleviates NAFLD via repressing FAS and

SCD1. Sci Rep. 2017;7:14493-502. 